Mereles, F., G. Cépedes, J. De Egea-Elsam \& R. Spichiger. 2020.

Estudios fitosociológicos en el gran Chaco: estructura, composición florística y variabilidad del bosque de Schinopsis balansae en el Chaco húmedo boreal, Paraguay. Bonplandia 29(1): 39-55.

Doi: http://dx.doi.org/10.30972/bon.2914108

Recibido 28 Mayo 2019. Aceptado 25 Septiembre 2019.

\title{
ESTUDIOS FITOSOCIOLÓGICOS EN EL GRAN CHACO: ESTRUCTURA, COMPOSICIÓN FLORÍSTICA Y VARIABILIDAD DEL BOSQUE DE SCHINOPSIS BALANSAE EN EL CHACO HÚMEDO BOREAL, PARAGUAY
}

\author{
Phytosociological studies in the Great Chaco: structure, floristic composition and variability of \\ the Schinopsis balansae forest in the humid boreal Chaco, Paraguay
}

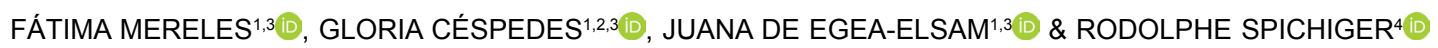

\begin{abstract}
Resumen: Se presenta la descripción del bosque de Schinopsis balansae que incluye un análisis de su estructura, composición y variabilidad florística. El sitio de muestreo se situó dentro del mosaico bosques-sabanas palmares en el Chaco húmedo. Se determinaron datos cuantitativos para el cálculo del Índice de Valor de Importancia, que incluyó la abundancia, frecuencia y dominancia de las especies. Se calculó el índice de Diversidad del mismo y el valor obtenido fue comparado con otros bosques de la región. Los resultados indican que el bosque es un complejo de Fabaceae-Anacardiaceae-Arecaceae, con asociaciones edafobotánicas muy características y que florísticamente se trata de un bosque mixto y transicional con otras formaciones vegetales de América del Sur.
\end{abstract}

Palabras clave: Bosque subhúmedo, Chaco, diversidad, estudios cuantitativos, Paraguay.

Summary: A description of the forest of Schinopsis balansae is presented, including an analysis of its structure, composition and floristic variability. The sampling site was located within the forest-palm savanna mosaic in the humid Chaco. Quantitative data was determined in order to calculate the Importance Value Index that included the abundance, frequency and dominance of the species. The Diversity Index was also calculated and compared with that of other forests in the region. Results indicate that this forest is a Fabaceae-Anacardiaceae-Arecaceae complex with very characteristic edapho-botanic associations, and that it floristically constitutes a mixed and transitional forest with other plant formations of South America.

Key words: Chaco, diversity, Paraguay, quantitative studies, subhumid forest.

\section{Introducción}

El bosque de Schinopsis balansae Engl., es una de las formaciones consideradas climácicas en el Chaco regional que comprende, en orden de tamaño al Chaco argentino (Ragonese et Castiglioni, 1970; Cabrera et Willink, 1980), paraguayo y boliviano (Morello et Adamoli,
1974). Se trata de una formación con mucho valor ecológico y también económico, tanto por la diversidad de especies leñosas y herbáceas que alberga, como por las extensiones que aún permanecen en el territorio. En el Paraguay hasta el momento de este trabajo, se carece de datos cuantitativos que puedan dar una idea de su estructura y composición florística real.

${ }^{1}$ Centro para el Desarrollo de la Investigación Científica (CEDIC). Fundación Moisés Bertoni (FMB) y Laboratorios Díaz-Gill. Manduvirá 635 C/ 15 de Agosto. E-mail: fmereleshaydar@gmail.com; juana.deegea@gmail.com

${ }^{2}$ Facultad de Ciencias Agrarias, Universidad Nacional de Concepción, Paraguay. E-mail: gloriacespedes@gmail.com

${ }^{3}$ Programa Nacional de Incentivo a Investigadores, Consejo Nacional de Ciencia y Tecnología, PRONII-CONACYT, Asunción.

${ }^{4}$ Conservatoire et Jardin botaniques, Ginebra, Suiza. E-mail: rodolphe.spichiger@ville-ge.ch 
Se distribuye en gran parte en el este de la región chaqueña, siguiendo el litoral del río Paraguay hasta el SE y ocupando la Mesopotamia entre los ríos Paraguay y Pilcomayo. La formación se desarrolla como parte del mosaico bosque-sabanas palmares (Ramella et Spichiger, 1989; Spichiger et al., 1991), al que se agregan los humedales en las partes más deprimidas (Mereles, 2005), desde las proximidades de la localidad de Fuerte

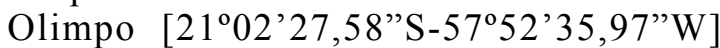
sobre el río Paraguay, hasta la proximidades

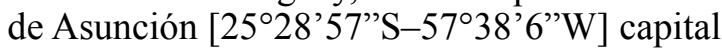
del país, bordeando por el $\mathrm{SO}$ del territorio chaqueño. Fitogeográficamente forma parte del Dominio Chaqueño, Provincia Chaqueña y Distrito Chaqueño Oriental (Cabrera et Willink, 1973). En el Chaco paraguayo, abarca dos de las cinco ecorregiones presentes en el territorio: Pantanal y Chaco húmedo (Mereles et al., 2013), (Fig. 1). La parte ocupada por el mosaico bosque-sabana palmar-humedales corresponde a la más húmeda del territorio chaqueño boreal, no solo debido a las precipitaciones más abundantes sino también a la cantidad de agua acumulada durante los periodos de aguas altas, a causa del alto contenido de arcilla en los suelos (Proyecto Sistema Ambiental del Chaco, suelos: 19921998).

En cuanto al clima, el bosque sigue aproximadamente los siguientes valores de temperatura media entre $10 \mathrm{~s} 23^{\circ} \mathrm{C}$ e isoyetas, las que van entre los 1000-1100 mm/año (Fig. 2).

Este bosque ha recibido diversas denominaciones, Fiebrig et Rojas (1933) lo incluyeron dentro de las formaciones xerofíticas del Chaco boreal; Tortorelli (1967) lo denominó como matorral xerófilo, agregando que se trata del bosque típico chaqueño asociado a suelos anegadizos y escasos en humus; bosque templado seco (Holdridge, 1967), atendiendo a la relación con el tipo de clima y sus componentes florísticos.

Otras denominaciones fueron bosque denso monoespecífico (Morello et Adámoli, 1974), en donde resaltaron la dominancia de Schinopsis balansae sobre las demás especies; bosque de quebracho colorado (Sanjurjo, 1977; Ramella et Spichiger, 1989), resaltando que se trata de un bosque climácico del
Chaco; Spichiger et al. (1991) lo mencionan como un bosque xeromesófilo con Schinopsis balansae y Astronium denominado quebrachal de quebracho colorado. Finalmente, Mereles (2005) lo menciona como bosque mesoxerófilo semicaducifolio, agregando a la mención de Spichiger et al. (1991) el carácter semideciduo de varios de sus componentes leñosos.

\section{Geomorfología, hidrología y suelos}

El paisaje chaqueño ha sido y sigue siendo modelado por uno de sus ríos más importantes, el Pilcomayo. Observaciones satelitales indican que este río antiguamente desembocaba en las cercanías de la localidad de Bahía Negra, [202 $\left.25^{\prime} \mathrm{S}-58^{\circ} 15^{\prime} \mathrm{W}\right]$, departamento Alto Paraguay, sitio desde donde se abre el gran abanico aluvial de los sedimentos del río, uno de los más grandes del mundo (Vera Morínigo et Palmieri, 1976), hasta la localidad de Salto Palmar [244ㅇ's-5058'W], departamento Presidente Hayes, salto que no está producido por ninguna falla geológica sino por una erosión retrocedente producida por la acumulación de los sedimentos aluvionales depositados en el cauce del río y que causan el desnivel en el suelo (Vera Morínigo, 1990). Las aguas del Pilcomayo procedentes del subsuelo, afloran dando lugar al salto. El bosque de $S$. balansae es una de las principales formaciones vegetales en medio de esta planicie sedimentaria.

Si bien la inclinación topográfica de la planicie chaqueña en general es de una pendiente aproximada de 575' (Vera Morínigo, 1990), ésta es bastante cambiante en superficies más pequeñas, por lo que su geomorfología es una de las grandes determinantes de las variantes de los tipos de vegetación existentes y por lo tanto, del paisaje (Ramella et Spichiger, 1989). En efecto, ante la mínima depresión y condicionada por los tipos de suelos existentes, el paisaje cambia completamente de una vegetación de aspecto xerófito y achaparrado a otra de aspecto más denso, inclusive de ambientes completamente terrestres a palustres y hasta acuáticos.

En la planicie chaqueña en Paraguay, el río Pilcomayo carece de tributarios y desemboca en el río Paraguay a través de brazos separados, conformando un delta interior o continental. En la región $\mathrm{NE}$ del territorio, quedan vestigios de 
F. Mereles et al., Estructura y variabilidad del bosque de Schinopsis balansae, Paraguay

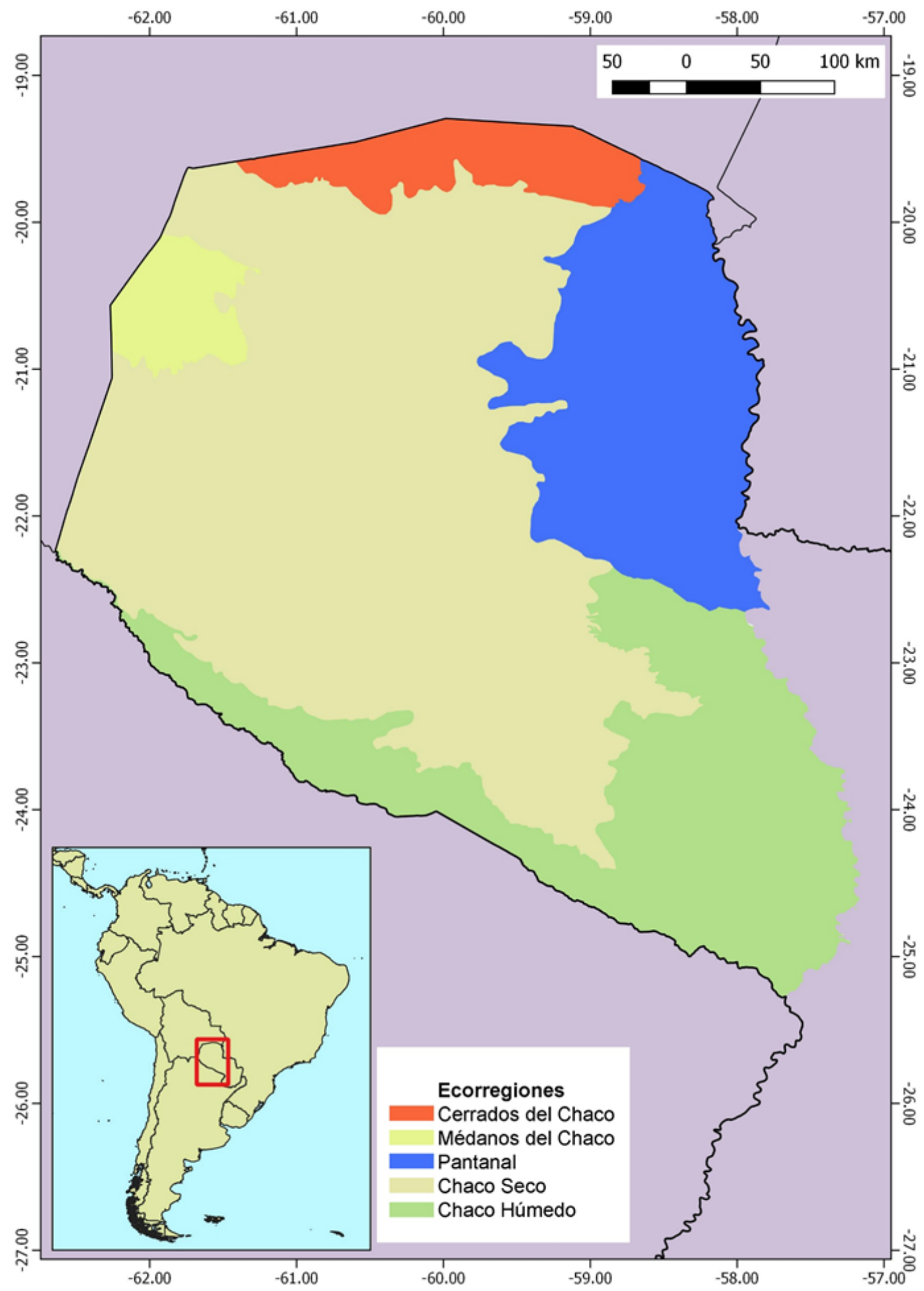

Fig. 1. Ecorregiones del Chaco paraguayo.

Fig. 1. Ecoregions of the Paraguayan Chaco. 


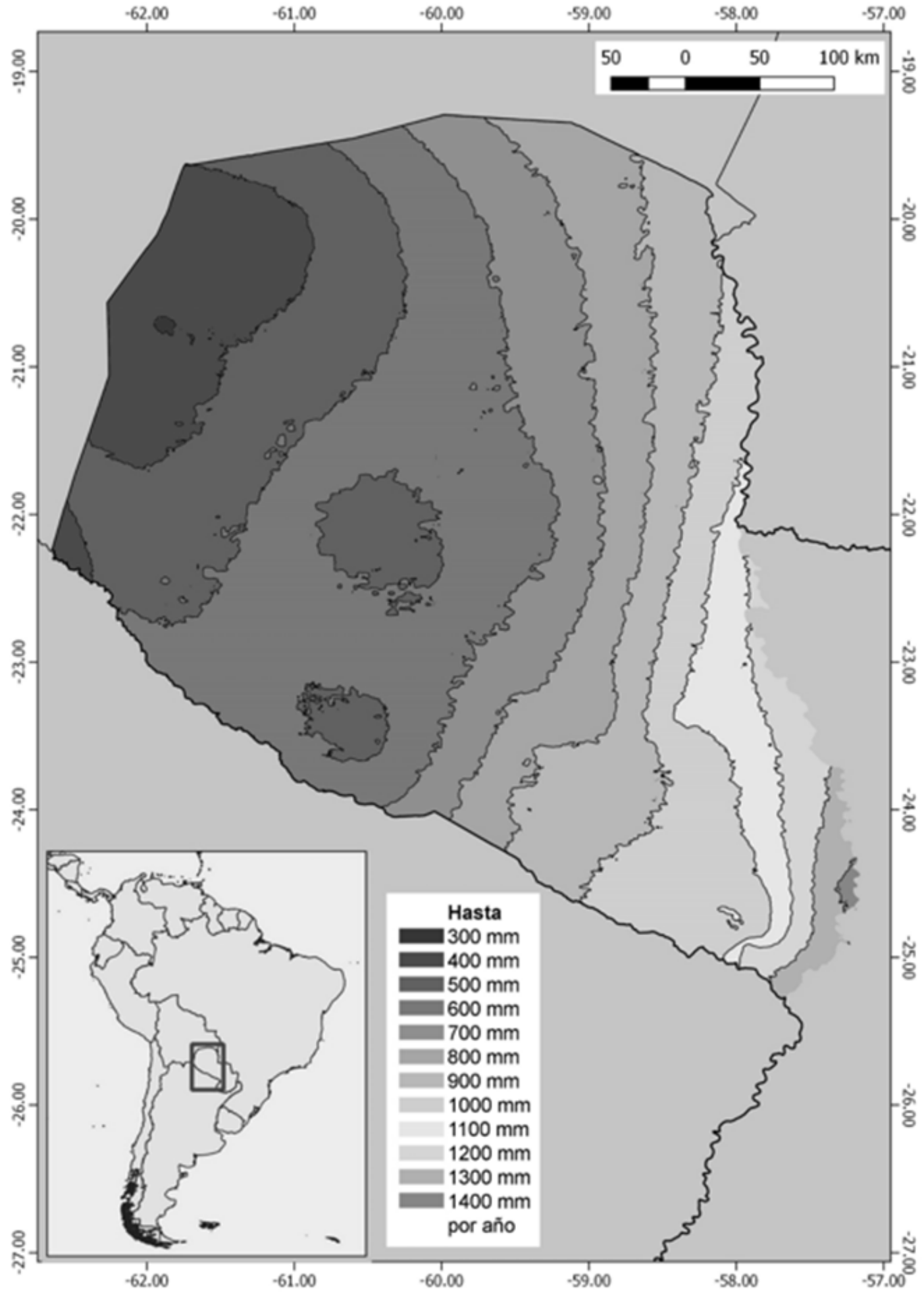

Fig. 2. Valores de isoyetas para el Chaco paraguayo.

Fig. 2. Isoyet values for the Paraguayan Chaco. 
los cursos de agua temporarios que conforman el antiguo delta interior y son los riachos Yacaré Norte y Yacaré Sur, Mosquito y González entre otros, que se entrecortan estacionalmente, por ello la denominación de "riachos" (Cordini, 1947).

Más al $\mathrm{S}$, en la mesopotamia ParaguayPilcomayo, los ríos son más activos todo el año, conformando el actual abanico fluvial o delta continental. Este último se inicia desde el río Verde, cuyo curso transporta la menor cantidad de agua respecto de los demás, siendo el brazo más nórdico del delta actual (Mariotti, 1927), con aguas bastante saladas inferiores a los $1 \mathrm{~m}$ en los últimos 30 años y variando de nivel acorde con las precipitaciones producidas dentro del área de influencia.

El Proyecto Sistema Ambiental del Chaco (1992-1998) menciona que otros ríos del delta actual son el río Siete Puntas, cuyo volumen de agua se nutre de las precipitaciones, manteniéndose casi siempre en nivel de subsuelo, excepto cuando las lluvias son abundantes. Otros ríos son el Negro, muy influenciado por los reflujos del río Paraguay en periodos de aguas altas, que penetran intensamente dentro del territorio. El Montelindo, con un régimen parecido al anterior, muy modificado debido a los frecuentes represamientos ilegales en las propiedades privadas más al oeste, con su consecuente aumento en la salinidad. El río Aguaray Guasú también muy influenciado por el reflujo del río Paraguay en periodos de aguas altas y finalmente el río Confuso, uno de los que arrastra mayor caudal de todos los brazos mencionados (Kysela, 1931).

Dado que el río Paraguay tiene su influencia desde la napa freática más elevada hacia el este, los bosques de $S$. balansae se inundan periódicamente a través de los tributarios o del mismo río Paraguay, según la ubicación pero al mismo tiempo se anegan a causa de las lluvias. Estos procesos de inundaciones $\mathrm{y}$ anegamientos son independientes unos de otros de manera que el agua está siempre presente en alguna parte del año, debido a las características de sus suelos y el régimen pluviométrico.

Finalmente hacia el S-SO de la planicie, el bosque de $S$. balansae se encuentra colindante con una gran sabana inundable/ anegable denominada Estero Patiño, otrora con bastante agua en superficie, con una vegetación dominada por leñosas de bajo porte y herbáceas anfibias en tiempos de sequía.

En cuanto al sustrato, los bosques de quebracho se desarrollan sobre suelos horizontales semipermeables, con buena disponibilidad de agua en superficie y relativamente buena escorrentía superficial a excepción de aquellos sitios en los que según la morfología, acumulen agua. Estos suelos se caracterizan por contener entre un $20-25 \%$ de sedimento arenoso, acompañados por un alto contenido en arcillas y limos, lo que los vuelve inundables y/o anegables por inundaciones y lluvias, muy estructurados y duros en seco, pegajosos cuando húmedos y salobres, del tipo planosoles (Proyecto Sistema Ambiental del Chaco, suelos: 1992-1998).

El trabajo tiene por objetivos realizar un perfil del mosaico de vegetación bosque-sabanas hidromórficas-humedales, analizar la estructura del bosque de $S$. balansae, determinar la densidad de árboles en una superficie definida, el Índice de Valor de Importancia (IVI), conocer su diversidad florística, correlacionar las existentes con otras masas boscosas en América del Sur y emitir recomendaciones acorde con el estado de conservación de los remanentes boscosos actuales.

\section{Material y Métodos}

\section{Selección del sitio de muestreo}

El sitio de muestreo se ubicó en la Estancia Santa María del Doce, retiro San

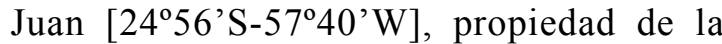
Fundación la Piedad, unos $40 \mathrm{~km}$ al $\mathrm{O}$ del río Paraguay, departamento Presidente Hayes, (Fig. 3). El mismo se seleccionó en base a las observaciones de las imágenes satelitales a escala 1:100.000 del tipo LANDSAT TM, lo que dio una primera aproximación de la distribución del mosaico de bosques al igual que el estado de conservación del mismo, ya que se trata de una zona ganadera. Otra razón fue la de tener acceso al sitio, considerando el mal estado de los caminos locales en periodos de lluvias. 


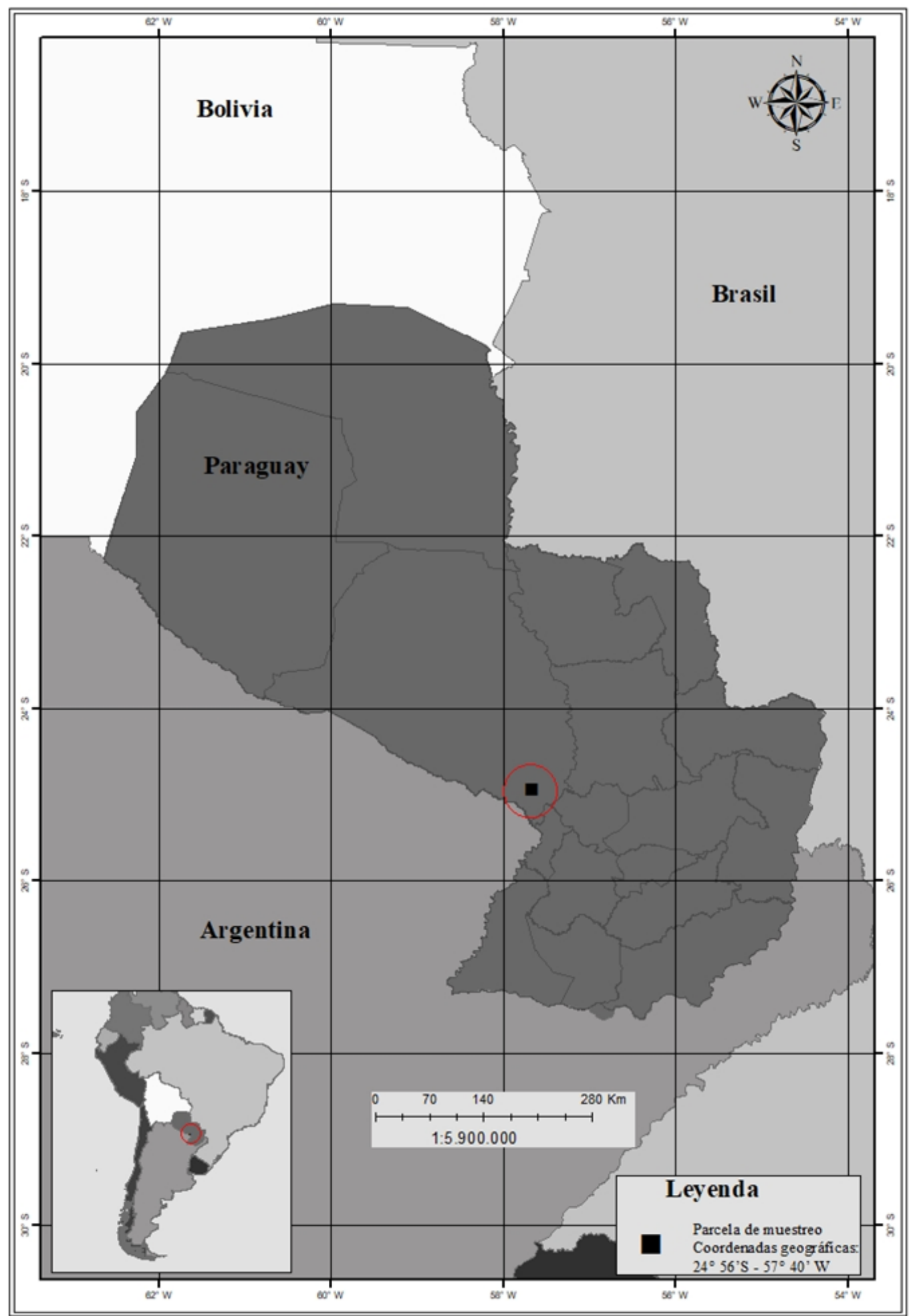

Fig. 3. Ubicación del sitio de muestreo.

Fig. 3. Location of the sampling site. 
Perfil del mosaico bosque-sabanas palmareshumedales y análisis estructural

El perfil del mosaico se realizó a través de las imágenes satelitales Landsat TM a escala 1:100.000 y posteriormente in situ, en recorridos sobre el terreno. El análisis estructural del bosque incluyó:

- Un perfil del bosque en función a la morfología del suelo, dentro del mosaico bosque-sabana palmar-humedales es decir ubicar a los componentes del mosaico (plantas acuáticas, palmares de Copernicia alba Morong y bosque) en el sitio que corresponde, acorde con la presencia de aguas permanentes y temporarias.

- La descripción fisonómica simple de la cobertura del bosque (Mateucci et Colma, 1982).

- Un análisis de la abundancia, frecuencia y dominancia de árboles por superficie (Lamprecht, 1990), siendo esta de 1 hectárea cuadrada, parcela mínima necesaria para un correcto análisis de la vegetación (Marmillod, 1982). La superficie fue subdividida en 25 subparcelas de $20 \times 20 \mathrm{~m}$ de lado cada una. Aunque el muestreo se realizó en una formación pura, aparentemente sin o con muy pocas afectaciones antrópicas, se trató de evitar el efecto borde penetrando al menos a unos $50 \mathrm{~m}$ al interior del bosque para el inicio de la construcción de la parcela.

La descripción fisonómico-estructural de la vegetación que proporciona el perfil, indica la disposición de las plantas de acuerdo a su forma de vida en los diferentes estratos, dependiente del tamaño y la ubicación de los sitios de muestreo.

La abundancia indica el número de individuos por cada especie encontrada en cada una de las subparcelas instaladas. La frecuencia da una idea aproximada de la homogeneidad del bosque y la dominancia es el grado de cobertura de las especies arbóreas, como una expresión del espacio ocupado y se define como la suma de las proyecciones horizontales de los árboles sobre el suelo. Debido a la estructura vertical complicada de los árboles en el trópico, su determinación es muy trabajosa (Lamprecht, 1990), por lo que se emplean las áreas basales como un sustituto de los verdaderos valores de la dominancia.
La densidad fue calculada en porcentaje de la suma total de la dominancia relativa, según la siguiente fórmula:

$$
\text { Dre }=B i / \sum B i \times 100
$$

Dre: dominancia relativa

Bi: área basal total de cada especie, calculada a partir de la medición del diámetro (Mateucci et Colma, 1982)

$\sum \mathrm{Bi}$ : sumatoria de las áreas basales de todas las especies

El área basal se calculó en base a la sumatoria del valor obtenido de cada individuo en función a su diámetro a la altura del pecho (DAP), los que fueron iguales o superiores a 10 cm (Lamprecht, 1990).

\section{Análisis florístico}

a) Leñosas

- Se relevaron las especies arbóreas del bosque, con las colectas del material de herbario para su determinación taxonómica, (riqueza en especies o diversidad alfa), Moreno (2001).

- El cálculo del Índice del Valor de Importancia (IVI), indica la preponderancia de las especies en el ecosistema, para lo cual se determinó la abundancia relativa $(\mathrm{A} \%)$, frecuencia relativa $(\mathrm{F} \%)$ y dominancia relativa $(\mathrm{D} \%)$, según la siguiente fórmula:

$$
\text { I. V.I }=\sum A \%+D \%+F \% \text {, siendo: }
$$

I.V.I: Índice del Valor de Importancia

A\%: Abundancia relativa

$\mathrm{D} \%$ : Dominancia relativa

F\%: Frecuencia relativa

El IVI es el mejor descriptor que cualquiera de los parámetros utilizados para catalogar individualmente a las especies (Mostacedo et Fredericksen, 2000) y cobra sentido si el objetivo es medir la diversidad biológica, pues aportan parámetros que permiten tomar decisiones o emitir recomendaciones a favor de la conservación de los taxones o áreas amenazadas. Igualmente permite definir florísticamente a los tipos de bosques.

b) Herbáceas del sotobosque

Se llevó un registro cualitativo de las herbáceas del sotobosque para conocer la 
cobertura herbácea. Las mismas fueron divididas de acuerdo a sus alturas en: aquellas que no sobrepasan los $20 \mathrm{~cm}$ (estrato herbáceo inferior), especies cuya altura es superior a 20 $\mathrm{cm}$ (estrato herbáceo superior) y especies cuya altura supera los 1,20 m (estrato subarbustivo). Se agregó un recuento de las lianas y epífitas. Posteriormente se procedió a la colecta de las especies fértiles.

Para la medición del Índice de Diversidad, se utilizó el índice de Shannon-Weaver, uno de los más comunes para determinar la diversidad de especies vegetales (Mostacedo et Fredericksen, 2000) que refleja la heterogeneidad de una comunidad sobre la base de dos factores: el número de especies presentes y su abundancia relativa (Pla, 2006). Los valores van del 1 al 5 , la diversidad es cero cuando solo existe una especie (Somarriba, 1999). El cálculo se basa en la siguiente fórmula:

$$
\mathrm{H}=-\sum \mathrm{Pi} * \ln \mathrm{Pi} .
$$

$\mathrm{H}=$ Índice de Shannon-Weaver

$\mathrm{Pi}=$ Abundancia relativa

$\ln =$ Logaritmo natural

\section{Análisis de los suelos}

Se realizaron determinaciones físicas de los tipos de suelos presentes al interior de la formación boscosa, a través de 4 calicatas de 1,50 $\mathrm{m}$ de profundidad en cada ángulo interior y una muestra con barrena en el centro. Las determinaciones fueron corroboradas por un equipo de especialistas edafólogos (Proyecto Sistema Ambiental del Chaco, suelos: 19921998). Estos se encuentran descriptos dentro del perfil del mosaico de vegetación.

\section{Resultados}

Perfil del mosaico bosque-sabanas palmareshumedales

El paisaje en donde prospera el bosque de S. balansae presenta un aspecto cambiante en pequeñas extensiones de terreno, debido a la geomorfología y los tipos de suelos. En la parte más elevada se desarrolla el bosque sobre suelos más profundos que dan lugar al desarrollo radicular de las leñosas. Éstos presentan un elevado contenido en arcillas con más del $40 \%$, bastante salobres e impermeables, anegables por lluvias e inundables por el desborde de los cauces de agua cercanos y en épocas de aguas altas puede llegar fácilmente a los 4-5 meses de anegamiento/inundación), del tipo planosoles gleycos.

En las partes más bajas se desarrollan los palmares de Copernicia alba, sobre suelos también ricos en arcillas, también salobres, impermeables e inundables por más tiempo que el anterior, llegando en periodos de aguas altas a 6-8 meses de anegamiento/inundación, del tipo gleysoles y vertisoles. Los palmares se caracterizan por la dominancia de la vegetación herbácea, bastante cambiante, dependiendo del grado de asfixia de los suelos, cuando permanecen anegados o inundados.

En las partes más deprimidas aparecen los humedales con agua permanente y/o cursos de agua que cortan la planicie, con dominancia de la vegetación acuático-palustre. En conjunto constituyen el mosaico de vegetación bosquesabana palmar-humedales (Fig. 4).

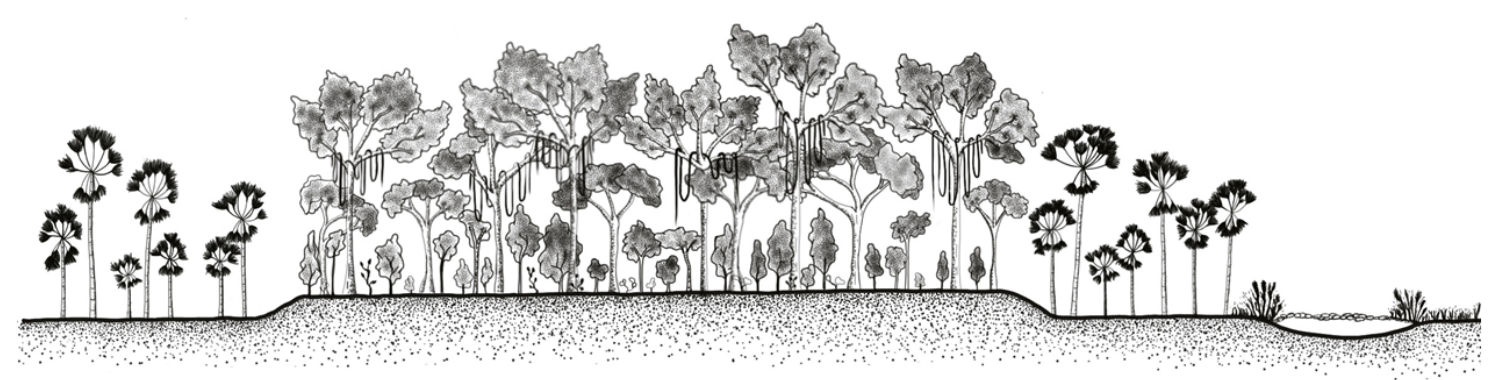

Fig. 4. Perfil del mosaico: bosque-sabanas palmares-humedales.

Fig. 4. Mosaic profile: forest-palm savannas-wetlands. 
Análisis estructural

Se trata de un bosque muy denso, con 4-5 estratos de vegetación en el que la estratificación no se ha podido visualizar claramente a partir de los doseles más elevados, aproximadamente $15 \mathrm{~m}$ en adelante. Sin embargo, se pudieron comprobar tres-cuatro estratos bien marcados, tres arbóreos y uno arbustivo, con abundantes lianas umbrófilas de tallo bastante grueso al interior de la masa y lianas heliófilas de tallo muy fino al exterior. La observación de la estratificación en el bosque de $S$. balansae si bien no ha sido clara en toda la superficie, sí fue visible en la mayor parte de la parcela estudiada.

Las herbáceas varían en altura hasta $1,30 \mathrm{~m}$, siendo característica la dominancia de Bromelia balansae Mez y Aechmea distichantha Lem. como tapiz herbáceo continuo y casi impenetrable en las áreas más abiertas. En cuanto a las alturas de las leñosas, las especies no sobrepasan los $25 \mathrm{~m}$ como máximo, arrojando los siguientes valores según los estratos, desde el primer dosel hacia abajo (Tabla 1).

Las especies del estrato herbáceo, en particular Aechmea distichantha y Bromelia balansae conforman un tapiz sin mezclarse entra ellas, $A$. distichantha a veces soporta inundaciones y $B$. balansae más al exterior, en sitios más abiertos, siempre secos. Las lianas y demás epífitas varían en su ubicación. Entre las lianas heliófilas, ubicadas siempre hacia el exterior de la formación boscosa, aparecen: Mikania cordifolia (L.f.) Willd., cuya ubicación coincide con los lugares más deprimidos y propensos a inundaciones, Fridericia dichotoma (Jacq.) L. G. Lohmann, Mascagnia brevifolia Griseb., Adenocalymma marginatum

Tabla 1. Resumen del número de individuos y sus alturas.

Table 1. Summary of the number of individuals and their heights.

\begin{tabular}{|c|ccccc|}
\hline Alturas & $20-25 m$ & $15-20 m$ & $10-15 m<10 m$ \\
\hline$N^{\circ}$ individuos & 57 & 110 & 138 & 91 \\
\hline
\end{tabular}

(Cham.) DC., Dolichandra unguis-cati (L.) L. G. Lohmann, Cardiospermum corindum L., Forsteronia glabrescens Müll. Arg., entre las más comunes. Otras como Aristolochia triangularis Cham., son umbrófilas.

Entre las epífitas ubicadas a menos de $5 \mathrm{~m}$ de altura se destacan Tillandsia meridionalis Baker, T. duratii Vis., T. loliacea Mart. ex Schult. f., Thaumatophyllum bipinnatifidum (Schott ex Endl.) Sakur, Calasanz \& Mayo, Oncidium pumilum Lindl. y Rhipsalis sp., entre otras.

La abundancia de individuos fue de 396. Las especies más abundantes fueron: $S$. balansae, Syagrus romanzoffiana (Cham.) Glassman, Libidibia paraguariensis (D. Parodi) J. P. Lewis, Gleditsia amorphoides (Griseb.) Taub., Astronium urundeuva Engl., Tabebuia nodosa (Griseb.) Griseb., Diplokeleba floribunda N. E. Br., Handroanthus heptaphyllus (Vell.) Mattos, Chloroleucon tenuiflorum (Benth.) Barneby \& J. W. Grimes, Calycophyllum multiflorum Griseb., Albizia inundata (Mart.) Barneby \& J. W. Grimes y Phyllostylon rhamnoides (J. Poiss.) Taub., seguidos de Pithecellobium scalare Griseb., Copernicia alba, y Albizia inundata (Tabla 2).

Respecto de las frecuencias relativas, $S$. balansae tiene la mejor distribución y le siguen: Syagrus romanzoffiana, Tabebuia nodosa, Astronium urundeuva, Libidibia paraguariensis, Handroanthus heptaphyllus, Gleditsia amorphoides, Diplokeleba floribunda, Albizia inundata, Phyllostylon rhamnoides y Chloroleucon tenuiflorum (Tabla 2).

Las 10 especies dominantes en el siguiente orden fueron: S. balansae, Handroanthus heptaphylluys, Chloroleucon tenuiflorum, Gleditsia amorphoides, Diplokeleba floribunda, Libidibia paraguariensis, Astronium urundeuva, Enterolobium contortisiliquum, Calycophyllum multiflorum y Syagrus romanzoffiana.

En cuanto a los diámetros (DIA) de los individuos, los resultados se reflejan en la Tabla 3. La clase diamétrica que suministra la mayor participación en la cobertura basal se encuentra entre los individuos que poseen entre 10 y $15 \mathrm{~cm}$ de DAP, en tanto que la de los árboles con $\mathrm{DAP} \geq 45 \mathrm{~cm}$, son relativamente 
Tabla 2. Especies más importantes del bosque según análisis de la estructura horizontal.

Table 2. Most important forest species according to horizontal structure analysis.

\begin{tabular}{|llccccc|}
\hline \multicolumn{1}{|c}{ Familia } & \multicolumn{1}{c}{ Especies } & $\begin{array}{c}\text { Abundancia } \\
\text { relativa }\end{array}$ & $\begin{array}{c}\text { Frecuencia } \\
\text { relativa }\end{array}$ & $\begin{array}{c}\text { Dominancia } \\
\text { relativa }\end{array}$ & I.V.I. \\
Anacardiaceae & Schinopsis balansae & 008 & 004 & 013 & 025 \\
Bignoniaceae & Handroanthus heptaphyllus & 005 & 003 & 009 & 017 \\
Fabaceae & Gleditsia amorphoides & 005 & 003 & 007 & 015 \\
Fabaceae & Libidibia paraguariensis & 005 & 003 & 005 & 013 \\
Sapindaceae & Diplokeleba floribunda & 004 & 003 & 006 & 013 \\
Fabaceae & Chloroleucon tenuiflorus & 003 & 003 & 006 & 013 \\
Anacardiaceae & Astronium urundeuva & 005 & 003 & 004 & 012 \\
Arecaceae & Syagrus romanzoffiana & 006 & 003 & 003 & 012 \\
Ulmaceae & Phyllostylon rhamnoides & 003 & 003 & 005 & 011 \\
\hline
\end{tabular}

escasos. De un total de 396 individuos, 362 poseen entre 10 y $25 \mathrm{~cm}$ de DAP. La relación entre el número de individuos y sus diámetros, se refleja en la Fig. 5.

Las especies con un DAP mayor a $45 \mathrm{~cm}$ fueron 11: Astronium urundeuva, S. balansae, Enterolobium contortisiliquum (Vell.) Morong, Gleditsia amorphoides, Anadenanthera colubrina (Vell.) Brenan var. cebil (Griseb.) Altschul, Peltophorum dubium (Spreng.) Taub., Calycophyllum multiflorum, Cordia americana (L.) Gottschling \& J. S. Mill. y Diplokeleba floribunda, entre otras.
Los valores de la Tabla 3 revelan que los árboles que poseen un diámetro entre $10 \mathrm{y}$ $25 \mathrm{~cm}$ son los más numerosos. Entre estos sobresalen Diplokeleba floribunda, Libidibia paraguariensis, Maytenus scutioides (Griseb.) Lourteig \& O'Donnel, Diatenopteryx sorbifolia Radlk., Alchornea triplinervia (Griseb.) Müll. Arg., Albizia inundata, Chloroleucon tenuiflorum, Chlorophora tinctoria (L.) Gaudich., Prosopis nigra (Griseb.) Hieron. y Phyllostylon rhamnoides.

Entre las leñosas con un DAP inferior o igual a $10 \mathrm{~cm}$, aparecen: Terminalia triflora

Tabla 3. Número de individuos según el tamaño de sus diámetros.

Table 3. Number of individuals according to the size of their diameters.

\begin{tabular}{|c|ccccc|}
\hline DAP & $\geq 10 \mathrm{~cm}$ & $\geq 15 \mathrm{~cm}$ & $\geq 25 \mathrm{~cm}$ & $\geq 45 \mathrm{~cm}$ & $\geq 60 \mathrm{~cm}$ \\
\hline $\mathbf{N}^{\circ}$ individuos & 285 & 77 & 23 & 7 & 4 \\
\hline
\end{tabular}


cant. ind

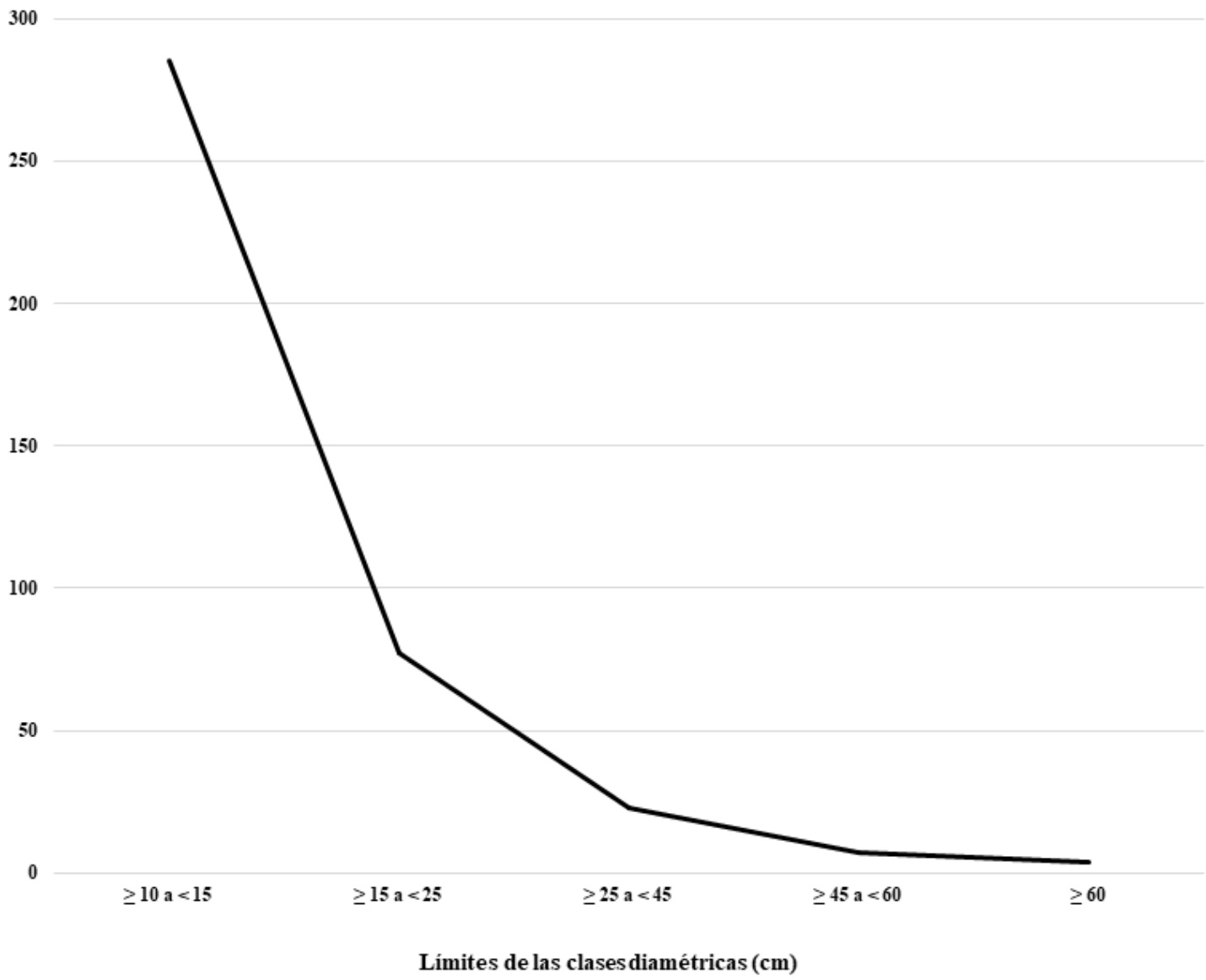

Fig. 5. Gráfico de la clase diamétrica.

Fig. 5. Diagram of the diameter class.

(Griseb.) Lillo, Phyllanthus chacoensis Morong, Inga vera Willd. subsp. affinis (DC.) T. D. Penn., Sorocea bonplandii (Baill.) W. C. Burger, Lanj. \& Wess. Boer, Crataeva tapia L., y Chrysophyllum marginatum (Hook. \& Arn.) Radlk., entre otras.

\section{Análisis florístico}

Se registraron 63 especies de leñosas pertenecientes a 59 géneros y 27 familias. Las especies con mayor importancia ecológica dentro del bosque fueron aquellas que dieron los mayores valores de Índice de Valor de Importancia, con un valor mayor a $10 \mathrm{y}$ fueron las siguientes: $S$. balansae (25\%),
Handroanthus heptaphyllus (17\%), Gleditsia amorphoides, Libidibia paraguariensis, Diplokeleba floribunda, Chloroleucon tenuiflorum, Astronium urundeuva, Syagrus romanzoffiana, Calycophyllum multiflorum y Phyllostylon rhamnoides. Como especies acompañantes fueron seleccionadas aquellas con valores entre 5 y 10 y fueron las siguientes: Tabebuia nodosa, Albizia inundata, Phyllostylon rhamnoides, Copernicia alba, Anadenanthera colubrina var. cebil, Enterolobium contortisiliquum, Microlobius foetidus (Jacq.) M. Sousa \& G. Andrade subsp. paraguensis (Benth.) M. Sousa \& G. Andrade, Cordia americana y Chlorophora tinctoria. 
En cuanto al Índice de Diversidad, la resultante $\mathrm{H}$ fue de 3,69 , por lo que puede considerarse a este bosque como medianamente diverso dentro de la escala de 1 a 5.

\section{Discusión}

Spichiger et al. (1991) clasifican al bosque de Schinopsis balansae dentro del contexto del mosaico de la flora del Chaco húmedo, el que se extiende a lo largo del litoral del río Paraguay e ingresa al interior de la planicie unos $60 \mathrm{~km}$ aproximadamente hasta llegar a la Mesopotamia Paraguay-Pilcomayo, (Mereles 2005). Según Placci (1995) y Lewis et al. (1997), comunidades muy similares aparecen en el Chaco argentino (provincias de Santa Fe y Formosa). Estas tres formaciones del mosaico constituyen un complejo muy ligado a los factores físicos dominantes como el agua superficial y atmosférica, el relieve superficial y los suelos. Dicho complejo se mantiene casi en forma permanente a lo largo de su extensión. Es de destacar que cada formación mantiene sus características propias. Acorde con Lewis et al. (1997), el bosque en sí puede definirse como subhúmedo y semicaducifolio, bastante denso en general aunque más abierto y discontinuo en algunos sitios, debido a la naturaleza de sus suelos muy arcillosos o porque algunos individuos leñosos se encuentran dañados.

Los siguientes componentes leñosos pierden sus hojas en forma parcial o totalmente: Peltophorum dubium, Enterolobium contortisiliquum, Handroanthus heptaphyllus, Libidibia paraguariensis, Astronium urundeuva, Gleditsia amorphoides, Calycophyllum multiflorum, Tabebuia nodosa, entre otras, sin que presente las características propias de los bosques estacionales propiamente dichos. Algunos componentes arbóreos como Libidibia paraguariensis, Cordia americana y $S$. balansae coinciden con los resultados obtenidos por Lewis et al. (1997).

Lo destacable es que dentro de tanta complejidad, estas formaciones mantienen su homogeneidad y la variabilidad depende del mínimo cambio de sus factores físicos, en particular el factor edáfico. En efecto, al analizar los componentes del bosque y los tipos de suelos dominantes, observamos que la vegetación boscosa conforma con estos, asociaciones edafobotánicas muy marcadas. En efecto, sobre los suelos más compactos, estructurados e impermeables del tipo gleysoles penetran algunas especies no típicas del bosque como el caso de Copernicia alba, el que se encuentra siempre en lugares inundables por más tiempo y probablemente donde ha llegado a penetrar el ganado vacuno, transportando las semillas, y donde se concentran sobre el mismo sustrato las siguientes especies: Calycophyllum multiflorum, Phyllostylon rhamnoides, Tabebuia nodosa, Prosopis nigra, P. hassleri Harms, Parkinsonia aculeata L. entre otras, especies propias de suelos anegables.

Otros grupos de especies se concentran sobre suelos arcillosos en los horizontes un poco más profundos y que presentan una capa arenosa en superficie, del tipo fluvisoles y son los que se encuentran en las cercanías del río Confuso y que probablemente en periodos de creciente arrastran las arenas y las depositan sobre la arcilla. Sobre estos suelos se concentran Nectandra megapotamica (Spreng.) Mez, Enterolobium contortisiliquum, Cynometra bauhinifolia Benth. var. bauhinifolia, Seguiera paraguayensis Morong, Chrysophyllum marginatum, Phyllanthus chacoensis, entre otras.

Un tercer grupo conformado por $S$. balansae, Astronium urundeuva, Libidibia paraguariensis, Diplokeleba floribunda, Anadenanthera colubrina var. cebil, Gleditsia amorphoides, Geoffroea decorticans (Gillies ex Hook. \& Arn.) Burkart, Syagrus romanzoffiana, Coccoloba sp. se concentran en mayor número en torno a los suelos del tipo planosoles, similares a los gleysoles pero con menor contenido de arcillas, aunque S. balansae, A. urundeuva, D. floribunda y $S$. romanzoffiana, Handroanthus heptaphyllus y Peltophorum dubium, se distribuyen bastante bien en casi todas las parcelas.

Finalmente, otras especies como Pterogyne nitens Tul., Meliccoccus lepidopetalus 
Radlk., Vachellia caven (Molina) Seigler \& Ebinger, Plinia rivularis (Cambess.) Rotman, Crataeva tapia y Parkinsonia aculeata, se ubican más hacia las borduras, siendo su presencia dentro del bosque casi nula. El caso de $M$. lepidopetalus es similar al de Copernicia alba cuyos frutos también hubieran sido transportados por el ganado, dado que es una especie que siempre acompaña al hombre, al igual que Pterogyne nitens.

Desde el punto de vista estructural, su estratificación corresponde a otros bosques existentes en el país, como los semihúmedos y húmedos semicaducifolios, con estratos bastante densos. Según Smith (1973), los bosques tropicales se organizan en estratos densos debido a la implicancia de numerosos factores abióticos que actúan sobre la fisiología de las especies que componen el bosque, tales como la luz, la concentración de $\mathrm{CO}_{2}$ y la humedad. Por otro lado, Richards et al. (1940), afirman que la estratificación en una selva tropical es difícil de discernirse probablemente debido a la densidad de los estratos y que la misma siempre existe, situación con la que acordamos para este caso. En coincidencia con Lewis et al. (1997), la cobertura de la vegetación leñosa en este bosque tampoco es de un $100 \%$ y el grado de superposición de los estratos tampoco es muy alto, lo cual significa que existen claros dentro del bosque e igualmente la capa arbustiva tampoco es continua, sino que se presentan claros. Consideramos que probablemente la razón fundamental son los suelos cambiantes de menos a más estructurados e impermeables en los claros o lo que Lewis et al. (1997) mencionan como "dinámicas sucesionales".

El análisis de la distribución de las clases diamétricas para las diferentes especies arbóreas, permite evaluar su estado ecológico y de conservación, detectar su estado de regeneración y el envejecimiento de la masa forestal. De hecho, la distribución de las clases diamétricas de una parte de un bosque refleja su estructura demográfica, por lo que las mismas fueron utilizadas para estudiar el dinamismo de las especies forestales, su relación con los factores ambientales y su respuesta a los diversos tipos de gestión (Bernardzki et al., 1998; Solomon et Gove, 1999). Si se observa la Fig. 5, ésta mantiene el patrón de la $\mathrm{J}$ invertida, lo que sugiere que el bosque en todos los bloques se regenera naturalmente y de manera favorable, ya que el número de individuos con menores clases es mayor, la vegetación se encuentra en un proceso de crecimiento continuo (Arruda et al., 2011; Imaña Encinas et al., 2011). Por lo tanto se puede inferir que este bosque se encuentra en pleno camino a la madurez y que la variación en los tipos de suelos encontrados al interior serían la causa de la distribución de las especies, puesto que no se han observado signos de modificaciones por tala de árboles sino únicamente por esporádicas entradas del ganado en la bordura. Los individuos con DAP mayores, desde $25 \mathrm{~cm}$ en adelante a más de $60 \mathrm{~cm}$ son 34 , lo que estaría corroborando las deducciones de un bosque ya estable, pero a su vez, en plena maduración.

La ausencia de ciertos diámetros podría deberse a épocas de crisis en la dinámica de la población forestal. En el caso estudiado, la razón de tanta diferencia numérica entre diámetros igual o menores a 10 , podría ser que individuos jóvenes pertenecientes a las clases diamétricas iguales o mayores a 45 $\mathrm{cm}$ pudieron haber sido consumidos por el ganado años atrás, o alguna inclemencia climática que los hubiera afectado, como por ejemplo un exceso de lluvias sobre suelos casi impermeables, situación también probable. Otra razón podría ser el hecho de que las tasas de crecimiento de algunos individuos de edad intermedia se hubieran visto afectadas por algunas condiciones ambientales, como por ejemplo la calidad del suelo y obviamente los factores genéticos de los individuos.

En un análisis de la distribución en el Paraguay del Dominio de los Bosques Secos Estacionales, Oakley et Prado (2011) llegan a la conclusión de que los bosques de $S$. balansae serían parte de este Dominio, mencionando la presencia en éste de especies tales como: Astronium urundeuva, Handroanthus heptaphyllus, Cordia americana, Terminalia triflora, 
Anadenantera colubrina var. cebil, Gleditsia amorphoides, Pterogyne nitens, Calycophyllum multiflorum y Phyllostylon rhamnoides. Sin embargo los autores no mencionan a $S$. balansae, ni a Syagrus romanzoffiana en el trabajo, especies prácticamente dominante en todo el Chaco húmedo y con uno de los mayores valores de importancia del bosque.

El análisis florístico destaca a las familias más representativas en cantidad y distribución, entre ellas Fabaceae (17 géneros y 23\% del total), Euphorbiaceae (4 géneros y 9,2\% del total) y Anacardiaceae y Sapindaceae (3 géneros y $4,6 \%$ del total, respectivamente).

En cuanto al Índice de Diversidad, concluimos que se trata de un bosque medianamente diverso (Moreno, 2001), mencionado por Torres-Torres et al. (2016). Comparando este índice con otros bosques (Tabla 4), puede deducirse que el bosque de $S$. balansae es tan diverso o más que otros bosques húmedos y semihúmedos de la región, excepto el bosque húmedo de la región Oriental del país (Torres, 2016). Lamentablemente con datos incompletos, según se menciona en dicha tabla (Tabla 4).

Tabla 4. Valores comparativos de Índice de Diversidad con otros bosques de la región.

Table 4. Comparative values of the Diversity Index with other forests in the region.

\begin{tabular}{|c|c|c|c|c|c|}
\hline Tipo de bosque & Autor & $\begin{array}{l}\text { Índice de } \\
\text { Shannon }\end{array}$ & $N^{\circ}$ spp. & $\begin{array}{c}\mathbf{N}^{\circ} \\
\text { géneros }\end{array}$ & $\begin{array}{c}N^{\circ} \\
\text { familias }\end{array}$ \\
\hline Bosque de Schinopsis balansae & $\begin{array}{l}\text { Mereles et al., } 2019 \text { (en } \\
\text { preparación) }\end{array}$ & 3,6 & 63 & 58 & 27 \\
\hline Bosque seco tropical & Fonseca et al. 2003 & 2,7 a 3,1 & 34 a 48 & 39 a 53 & 23 a 32 \\
\hline Bosque seco/Ecuador & $\begin{array}{l}\text { Aguirre Mendoza et al. } \\
2013\end{array}$ & 2,38 a 2,82 & 58 & 51 & 29 \\
\hline Bosque seco/Ecuador & Muñoz et al., 2014 & 2,11 a 2,51 & 21 & sin datos & 14 \\
\hline Bosque seco/Panamá & López et al., 2015 & 3,13 & 52 & sin datos & 25 \\
\hline Bosque chaqueño & Giménez et al., 2011 & 0,63 a 2,07 & 46 & sin datos & 20 \\
\hline Bosque Chaco serrano & $\begin{array}{l}\text { Hernández et Giménez, } \\
2016\end{array}$ & 2,65 & 63 & 44 & 23 \\
\hline Bosque Chaco serrano & Varela et al., 2002 & 2,30 a 2,97 & 27 & sin datos & 16 \\
\hline Cerrado de la Chiquitanía & Villarroel et al., 2010 & 1,5 & 12 & 10 & 8 \\
\hline Bosque húmedo/Costa Rica & $\begin{array}{l}\text { Cascante et Estrada, } \\
2001\end{array}$ & 2,9 a 3,5 & 106 & 83 & 40 \\
\hline $\begin{array}{l}\text { Bosque húmedo tropical/ } \\
\text { Colombia }\end{array}$ & Torres-Torres et al., 2016 & 3 a 3,28 & 83 & 66 & 32 \\
\hline $\begin{array}{l}\text { Bosque húmedo de la región } \\
\text { oriental/Paraguay }\end{array}$ & Torres, M. 2016 & 4,23 & $\begin{array}{c}\sin \\
\text { datos }\end{array}$ & sin datos & sin datos \\
\hline
\end{tabular}




\section{Conclusiones y Recomendación}

En cuanto a su distribución podemos afirmar que el bosque se trata de un complejo de Fabaceae-Anacardiaceae-Arecaceae, por los altos valores de IVI de algunas especies de las familias mencionadas. Podemos afirmar en base a los resultados que a) las especies componentes del bosque conforman asociaciones edafobotánicas muy marcadas, b) que el bosque actual es típicamente una formación mixta y transicional entre las formaciones boscosas de las cuencas de los ríos Paraguay, Paraná, piedemonte andino, borde de la Amazonía, Cerrado y Caatinga, pues sus componentes son autóctonos de la región chaqueña y piedemonte andino $(S$. balansae, Libidibia paraguariensis, Prosopis nigra, Phyllanthus chacoensis, Microlobius foetidus subsp. paraguensis, Calycophyllum multiflorum y Tabebuia nodosa) y alóctonos de otras regiones como la cuenca del río Paraguay (Diplokeleba floribunda, Diatenopteryx sorbifolia, Handroanthus heptaphyllus, Cordia americana, Peltophorum dubium, Apuleia leiocarpa (Vogel) J. F. Macbr., Trichilia elegans A. Juss. y Syagrus romanzoffiana, entre otras), o provenientes de la cuenca del río Paraná (Anadenanthera colubrina y Gleditsia amorphoides, Stutz de Ortega (1983), también provenientes dentro del contexto de Residual Pleistocenic Dry Seasonal Flora, ubicado entre la cuenca amazónica y los campos cerrados (Spichiger et al., 1995), y finalmente la caatinga y cerrados con Astronium urundeuva, Prado et Gibbs (1993), como también dentro del contexto de las RPD Flora (Spichiger et al., 1995).

Si bien estas formaciones boscosas se encuentran en buen estado de conservación en general, las ubicadas hacia el extremo noreste del Chaco paraguayo pueden estar en serio peligro de alta fragmentación, por los índices elevados de deforestación registrados en la actualidad en el territorio. Se recomienda evaluar periódicamente la continuidad de los mismos y en caso de discontinuidad, aplicar ciertas herramientas de conservación como por ejemplo los corredores ecológicos u otro tipo de conectividades que puedan mantener con resiliencia los remanentes boscosos.

\section{Agradecimientos}

Los autores agradecen al Departamento de Botánica, Dirección de Investigación de la Facultad de Ciencias Químicas, Universidad Nacional de Asunción, por el apoyo para la realización de esta investigación.

\section{Bibliografía}

AGUIRRE MENDOZA, Z., Y. BETANCOURT FUGUERAS, G. GEADA LÓPEZ \& H. JASEN GONZÁLEZ. (2013). Composición florística, estructura de los bosques secos y su gestión para el desarrollo de la provincia de Loja, Ecuador. Avances 15: 144-155.

ARRUDA, D. M., D. O. BRANDAO, F. V. COSTA, G. S. TOLENTINO, R. D. BRASIL, S. N. D'ANGELONETO \& R. NUNES FERREIRA. (2011). Structural aspect and similarity among tropical dry forest fragments with different management histories in Northern Minas Gerais, Brazil. Arvore 35: 131-142. https://doi.org/10.1590/S0100-67622011000100016

BERNARDZKI, E., L. BOLIBOK, E. BRZEZIECKI, J. ZAJACZCKOWSKI \& H. ZYBURA. (1998). Compositional dynamics of natural forest in the Bialowieza National Park, Poland. Journal of Vegetation Science 9: 229-236. https://doi.org/10.2307/3237122

CABRERA, A. L. \& A. WILLINK. (1980). La vegetación de Paraguay en el cuadro fitogeográfico de América del Sur. Bol. Sociedad Argentina de Botánica 11, suplemento: 133-160.

CABRERA, A. L. \& A. WILLINK. (1973). Biogeografía de América Latina. Monografías Científicas serie biológica 13. Organización de Estados Americanos, O. E. A., Washington D. C. 122 pp.

CASCANTE, A. \& A. ESTRADA. (2001). Composición florística y estructura de un bosque húmedo premontano en el Valle Central de Costa Rica. Revista de Biología Tropical 49: 1-12.

CORDINI, R. (1947). Los ríos Pilcomayo en la región del Patiño. Anales de la Dirección de Minas y Geología de Argentina 1: 1-83.

FIEBRIG, K. \& T. ROJAS. (1933). Ensayo fitogeográfico del Chaco boreal. Revista del Jardín Botánico y Museo de Historia Natural del Paraguay 3: 3-87.

FONSECA G., W. E. CHAVÉ, CH. F. MORA \& P. V. MEZA. (2003). Dinámica y Composición del Bosque Seco Tropical. Resumen del XII Congreso 
Forestal Mundial, Quebec, Canadá. Consultado 10/05/2017: http://www.fao.org/docrep/ARTICLE/ WFC/XII/0203B4.HTM

GIMÉNEZ, A. M., P. HERNÁNDEZ, M. E. FIGUERO \& I. BARRIONUEVO. (2011). Diversidad del estrato arbóreo en los bosques del Chaco Semiárido. Quebracho 19: 24-37.

HERNÁNDEZ, P. \& A. M. GIMÉNEZ. (2016). Diversidad, composición florística y estructura en el Chaco serrano, Argentina. Madera y Bosques 22: 37-48. https://doi. org/10.21829/myb.2016.2231455

HOLDRIDGE, L. (1967). Ecología basada en zonas de vida. Instituto Latinoamericano de Ciencias Agrícolas (IICA), San José, Costa Rica. 216 pp.

IMAÑA ENCINAS, J., O. ANTUNEZ SANTANA \& C. RAINIER IMAÑA. (2011). Estructura diamétrica de un fragmento del bosque tropical seco de la región del Eco-Museo del Cerrado, Brasil. Colombia Forestal 14: 23-30.

https://doi.org/10.14483/udistrital.jour.colomb. for.2011.1.a02

KYSELA, V. (1931). El régimen hidráulico del río Confuso, Chaco paraguayo y sus repetidos cambios parciales de cauce. Revista de la Sociedad Científica del Paraguay $3: 37$.

LAMPRECHT, H. (1990). Silvicultura en los trópicos: los ecosistemas forestales en los bosques tropicales y sus especies arbóreas: posibilidades y métodos para un aprovechamiento sostenido. Cooperación Técnica Alemana (GTZ), Eschborn. 335 pp.

LEWIS, J. P., E. F. PIRE \& I. M. BARBERIS. (1997). Structure, physiognomy and floristic composition of a Schinopsis balansae (Anacardiaceae) forest in the Southern Chaco, Argentina. Revista de Biología Tropical 45: 1013-1020.

LÓPEZ, O. R., R. PÉREZ \& E. MARISCAL. (2015). Diversidad de árboles y arbustos en fragmentos de bosque seco tropical en Rio Hato, Panamá. Colombia Forestal 18: 105-115.

https://doi.org/10.14483/udistrital.jour.colomb. for.2015.1.a06

MARIOTTI, M. (1927). Sobre algunos deltas de ríos paraguayos. Revista de la Sociedad Científica del Paraguay 2: 148-149.

MARMILLOD, D. (1982). Methodik und Ergebnisse von Untersuchungen uber Zuzammensentzung und Aufbau eines Terrassenwaldes im peruanischem Amazonien. Diss. Goettingen. $67 \mathrm{pp}$.

MATTEUCCI, S. \& A. COLMA. (1982). Metodología para el estudio de la vegetación. Secretaría General de la Organización de Estados Americanos, O.E.A.,
Programa Regional de Desarrollo Científico y Tecnológico. Washington D.C. 168 pp.

MERELES, F. (2005). Una aproximación al conocimiento de las formaciones vegetales del Chaco paraguayo. Rojasiana 6: 5-48.

MERELES, F., J. L. CARTES, R. P. CLAY, P. CACIALI, C. PARADEDA \& A. YANOSKY. (2013). Análisis cualitativo para la definición de las ecorregiones de Paraguay Occidental. Paraquaria Natural 1: 12-20.

MORELLO, J. \& J. ADÁMOLI. (1974). La vegetación de la República Argentina: las grandes unidades de vegetación y ambientes del Chaco argentino II: vegetación y ambiente en la provincia del Chaco. Instituto Nacional de Tecnología Agropecuaria (INTA), ser. Fitogeográfica 13: 40-45.

MORENO, C. E. (2001). Métodos para medir la biodiversidad. M \& T Manuales y Tesis SEA, vol. 1. Zaragoza, España. 148 pp.

MOSTACEDO, B. \& B. FREDERICKSEN. (2000). Manual de Métodos Básicos de Muestreo y Análisis en Ecología Vegetal. Bolivia Forestal (BOLFOR), Santa Cruz, Bolivia. $87 \mathrm{pp}$.

MUÑOZ, J., ERAZO, S. \& D. ARMIJOS. (2014). Composición florística y estructura del bosque seco de la quinta experimental "El Chilco" en el suroccidente del Ecuador. CEDAMAZ 4: 53-61.

OAKLEY, L. \& D. PRADO. (2011). El dominio de los bosques secos estacionales neotropicales y la presencia del arco pleistocénico en la República del Paraguay. Rojasiana 10: 55-75.

PLA, L. (2006). Biodiversidad: Inferencia basada en el índice de Shannon y la riqueza. INTERCIENCIA 31: 583-590.

PLACCI, L. (1995). Estructura y comportamiento fenológico en relación a un gradiente hídrico en bosques del este de Formosa. Tesis Doctoral. Facultad de Naturales, Universidad Nacional La Plata, Argentina. 169 pp.

PRADO, D. \& P. GIBBS. (1993). Patterns of species distribution in the dry seasonal forest of South America. Annals of the Missouri Botanical Garden 80: 902-927. https://doi.org/10.2307/2399937

PROYECTO SISTEMA AMBIENTAL DEL CHACO. (1992-1998). Informe Técnico: suelos. Instituto Federal de Geociencias y Recursos Naturales (BGR), Alemania y Dirección de Ordenamiento Ambiental, Ministerio de Agricultura y Ganadería (DOA-MAG), Paraguay.

RAGONESE, A. E. \& J. C. CASTIGLIONI. (1970). La vegetación del Parque Chaqueño. Boletín de la Sociedad Argentina de Botánica 11 (Suplemento): 133-160. 
RAMELLA, L. \& R. SPICHIGER. (1989). Interpretación preliminar del medio físico y de la vegetación del Chaco boreal, Paraguay. Contribución al estudio de la flora y la vegetación del Chaco. I. Candollea 44: 639-680.

RICHARDS, P. W., A. G. TANSLEY \& A. S. WATT. (1940). The recording of structure, life forms and flora of tropical forest communities as a basis for their classification. Journal of Ecology 28: 224-239. https://doi.org/10.2307/2256171

SANJURJO, M. (1977). Proyecto Biológico Forestal. I. Estudio de algunos aspectos forestales. Organización de Estados Americanos (OEA) e Instituto de Ciencias Básicas, Universidad Nacional de Asunción (UNA).

SMITH, A. P. (1973). Stratification of temperate and tropical forest. The American Naturalist 107: 671683. https://doi.org/10.1086/282866

SOLOMON, D. S. \& J. H. GOVE. (1999). Effects of uneven-age management intensity on structural diversity in two major forest types in New England. Forest Ecology and Management 114: 265-274. https://doi.org/10.1016/S0378-1127(98)00358-2

SOMARRIBA, E. (1999). Diversidad Shannon. Agroforestería en las Américas 6: 72-74.

SPICHIGER, R., L. RAMELLA, R. PALESE \& F. MERELES. (1991). Proposición de leyenda para la cartografía de las formaciones vegetales del Chaco paraguayo. III. Candollea 46: 541-564.

SPICHIGER, R., R. PALESE, A. CHAUTEMS \& L. RAMELLA. (1995). Origin, affinities and diversity hot spots of the Paraguayan dendrofloras. Candollea 50: 517-537.

STUTZ DE ORTEGA. (1983). Etudes floristiques de divers etades secondaires des formations forestieres de Haut
Paraná (Paraguay Oriental). Inventaire floristiques d'une reserve forestiere. Candollea 38: 542-573.

TORRES, M. (2016). Los retos de conservación en el del Bosque Atlántico y los esfuerzos del INFONA. Instituto Forestal Nacional. Paraguay. 32 pp.

TORRES-TORRES, J. J., V. E. MENA MOSQUERA \& E. ÁlVAREZ-DÁVILA. (2016). Composición y diversidad florística de tres bosques húmedos tropicales de edades diferentes en el Jardín Botánico del Pacífico, municipio de Bahía Solano, Chocó, Colombia. Revista Biodiversidad Neotropical 6: 12-21. https://doi.org/10.18636/bioneotropical. v6il.197

TORTORELLI, L. (1967). Formaciones forestales y maderas del Paraguay. Boletín del Instituto Forestal Latinoamericano de Investigaciones 24: 3-34.

VARELA, R. O., E. ROSSI DE CEBALLOS, M. O. SIDÁN \& T. C. PERERA. (2002). Estructura, diversidad y relaciones florísticas de un bosque chaqueño serrano de Argentina. Contribución al estudio de la flora y de la vegetación del Chaco. XIV. Candollea 57: 239-249.

VERA MORÍNIGO, G. \& J. H. PALMIERI. (1976). Contribución al conocimiento de la hidrogeología de la zona oeste del Chaco paraguayo. Informes Técnicos, ser. D, 16. Instituto de Ciencias Básicas, Universidad Nacional de Asunción (UNA).

VERA MORÍNIGO, G. (1990). Aguas subterráneas: su importancia y preservación. La Revista Crítica 1: 67-72.

VILLAROEL, D., J. C. CATARI, D. CALDERÓN, R. MENDEZ \& T. FELDPAUSCH. (2010). Estructura, composición y diversidad arbórea de dos áreas de Cerrado sensu stricto de la Chiquitanía (Santa Cruz, Bolivia). Ecología en Bolivia 45: 116-130. 
